\title{
Perfil e percepção de profissionais dos Centros de Especialidades Odontológicas do Rio Grande do Sul
}

\author{
Profile and perception of professionals of Dental Specialty Centers \\ in the state of Rio Grande do Sul, Brazil
}

Karla Frichembruder ${ }^{*}$

Fernando Neves Hugo**

Juliana Balbinot Hilgert ${ }^{* * *}$

\section{Resumo}

Objetivo: traçar o perfil e descrever a percepção dos profissionais sobre condições e relações de trabalho e $o$ grau de satisfação ao atuar no Centro de Especialidades do Rio Grande do Sul. Sujeitos e método: 108 profissionais de 17 Centros de Especialidades Odontológicas do Rio Grande do Sul participaram do estudo e responderam a um questionário estruturado. Resultados: os resultados revelaram a presença de um grupo equilibradamente distribuído segundo o sexo, com idade média de 41,9 anos $( \pm 11,2)$, predomínio de etnia branca, alta frequência de pós-graduados $(81,5 \%)$ e maior proporção de vínculo empregatício formal $(67,6 \%)$. A maioria dos elementos relativos às condições e relações de trabalho foi julgada positivamente, com exceção do aspecto salarial e do dimensionamento da equipe de auxiliares em saúde bucal. O grau de satisfação com o trabalho nos Centros de Especialidades Odontológicas foi alto. Conclusão: os resultados apontam para uma relação positiva entre os profissionais e destes com seu ambiente de trabalho. Os elementos de maior fragilidade foram: condições de vinculação de trabalho no interior do Estado, condições salariais, atividades de capacitação, ações de educação permanente e o dimensionamento da equipe de pessoal auxiliar.

Palavras chave: Atenção secundária à saúde. Serviços de saúde. Recursos humanos em saúde.

\section{Introdução}

A organização da rede de atenção à saúde bucal sofreu impulso e transformação com a incorporação da equipe de saúde bucal na Estratégia de Saúde da Família (ESF) em 2000, e com o incentivo à implantação dos Centros de Especialidades Odontológicas (CEO) em 2004. A ampliação da cobertura de serviços de atenção básica e especializada ocorreu em todo o Brasil. Em 2010, eram 832 CEOs no país, hoje já são mais de 1000 serviços $^{1}$.

Tendo a atenção básica como coordenadora do cuidado, espera-se que o CEO esteja integrado ao processo de planejamento loco-regional, e que oferte, de acordo com a realidade epidemiológica de cada região, procedimentos clínicos odontológicos complementares aos realizados na atenção básica, seguindo a diretriz da integralidade ${ }^{2}$. Entre as definições das portarias que regulamentam o CEO destaca-se a demarcação de atividades mínimas obrigatórias: atenção especializada em cirurgia oral menor, endodontia, periodontia, diagnóstico bucal com ênfase no diagnóstico e detecção do câncer bucal e atenção aos pacientes portadores de necessidades especiais, e de um número mínimo de procedimentos para cada atividade. A equipe foi dimensionada para uma estrutura física definida que pode apre-

\footnotetext{
Doutoranda, Programa de Pós-Graduação em Odontologia, Faculdade de Odontologia, Universidade Federal do Rio Grande do Sul, Porto Alegre, RS, Brasil. Diretor do Centro de Pesquisas em Odontologia Social, Faculdade de Odontologia, Universidade Federal do Rio Grande do Sul, Porto Alegre, RS, Brasil. Professor do Programa de Pós-Graduação em Odontologia, Faculdade de Odontologia, Universidade Federal do Rio Grande do Sul, Porto Alegre, RS, Brasil. *** Professora do Programa de Pós-Graduação em Epidemiologia da Faculdade de Medicina e do Programa de Pós-Graduação em Odontologia, Faculdade de Odontologia, Universidade Federal do Rio Grande do Sul, Porto Alegre, RS, Brasil.
} 
sentar três portes que se constituem nas modalidades 1,2 e $3^{3,4,5}$. O repasse dos recursos financeiros está atrelado ao alcance de metas mínimas estabelecidas segundo a modalidade e as atividades obrigatórias definidas em portarias ${ }^{6,7,8}$.

É crescente o consenso entre gestores e profissionais de que a formação, o desempenho, e a gestão de recursos humanos afetam a qualidade dos serviços prestados e o grau de satisfação dos usuários ${ }^{9}$. Considerando a escassez de informações sobre os profissionais do CEO, elemento chave nesta nova organização, este estudo buscou conhecer o perfil dos coordenadores e cirurgiões-dentistas que atuam nos CEOs do Rio Grande do Sul (RS), sua percepção sobre as condições e relações de trabalho, e o grau de satisfação.

\section{Sujeitos e método}

Este é um estudo do tipo descritivo, aprovado pelo Comitê de Ética em Pesquisa do Grupo Hospitalar Conceição, segundo parecer n⿳ำ 10-169.

Os critérios de elegibilidade para participação foram: o CEO do RS estar habilitado pelo MS e em funcionamento, e para os profissionais, possuir vínculo trabalhista. Dos vinte CEOs que estavam habilitados pelo MS em 2010, um foi inelegível por não estar funcionando e dois foram perdidos por não devolução do Termo de Consentimento da Instituição. Os profissionais de $17 \mathrm{CEOs}$ do RS consentiram em participar através do Termo de Consentimento Livre e Esclarecido.

Foi utilizado um questionário composto por perguntas abertas e fechadas. Parte do questionário incluiu questões originais ou adaptações do questionário do Programa Nacional de Avaliação dos Serviços de Saúde ${ }^{10}$. Para os coordenadores foram incluídas questões específicas de características do CEO, agrupadas segundo as variáveis: modalidade, tempo de habilitação, área de abrangência, reuniões de equipe, reuniões com a Atenção Primária em Saúde (APS) e recebimento de estagiários de Odontologia.

$\mathrm{O}$ perfil do profissional foi traçado segundo as variáveis: sexo, idade, etnia, instituição e tempo de formação e pós-graduação. As variáveis atribuídas para caracterizar as condições e relações de trabalho no CEO são: tipo e tempo de vínculo com a instituição e com o CEO, renda, benefícios, ocupação, carga horária, conhecimento e exigência de metas de produção, número de pacientes atendidos, trabalho a quatro mãos com pessoal auxiliar e com cirurgião-dentista (CD), acesso a outros profissionais para discussão, protocolos e educação permanente.

A avaliação dos componentes estruturais e relacionais do CEO foi obtida através de questões como "como você julga": o tamanho da equipe (dimensionamento) para diferentes funções, a segurança para execução do trabalho, o ambiente físico, a disponibilidade de equipamentos e materiais, a higiene do ambiente, o salário, a carga de trabalho, o relacionamento com colegas, o relacionamento com chefia imediata e a valorização de seu trabalho pelo serviço, apresentadas numa escala tipo Likert: muito bom, bom, regular, ruim, muito ruim. Para fins de análise estatística, as variáveis foram recategorizadas em "bom (muito bom e bom), regular, e ruim (muito ruim e ruim)".

As respostas das questões abertas, como áreas de titulação de pós-graduação, motivos referidos para considerar o CEO um local privilegiado para atuar em relação à APS e problemas de saúde, foram analisadas e agrupadas por semelhança.

A satisfação do profissional com o trabalho no CEO foi representada pela pergunta "que grau de satisfação você atribui ao seu trabalho no CEO”, numa escala Lickert em que a resposta variou de muito baixo a muito alto. As respostas foram dicotomizadas em "alto" (alto e muito alto) e "baixo" (regular, baixo e muito baixo), devido à baixa ocorrência de baixo e muito baixo.

Foram realizadas frequências absolutas e relativas para as variáveis qualitativas e, média, desvio-padrão, mediana e percentis para as variáveis quantitativas. As análises de frequências, e as tabelas de contingência foram realizadas no software para análise estatística PASW Statistics 18 (National Defunct Lab, USA).

A logística da coleta obedeceu às seguintes fases: coleta por meio eletrônico, via FormSUS, com acesso ao questionário após o aceite do TCLE, disponibilizado por 30 dias. Os não respondentes foram contatados a cada fase. $\mathrm{O}$ número de questionários retornados foi de 110 (90,2\%), sendo que 108 aceitaram participar $(88,5 \%)$.

\section{Resultados}

Dos 17 CEOs estudados, a maioria era de modalidade tipo 1 (68,4\%), com habilitação há mais de 48 meses $(73,7 \%)$ e de abrangência municipal (73,3\%). As reuniões de equipe ocorrem em $86,7 \%$ dos serviços, com frequência mensal $(53,8 \%)$, a maioria dos CEOs (80,0\%) participava de reuniões com a APS. Nenhum CEO tipo 3 foi encontrado.

A Tabela 1 exibe o perfil dos profissionais. Observou-se um equilíbrio entre o número de profissionais segundo o sexo, predomínio da etnia branca, sendo a faixa etária de maior representação a de 30 a 39 anos (34,6\%). A titulação de doutorado teve forte representação no CEO universitário (53,8\% de doutores), sendo que seis CEOs (40,0\%) recebiam estagiários de Odontologia. 
Tabela 1 - Características sócio-demográficas dos profissionais, CEOs, RS, 2010

\begin{tabular}{|c|c|c|c|}
\hline $\mathrm{Va}$ & iável & $\begin{array}{c}\text { Coordenadores } \\
\mathrm{N} / \%(\mathrm{~N}=15)\end{array}$ & $\begin{array}{l}\text { Dentistas } \\
\mathrm{N} / \%(\mathrm{~N}=93)\end{array}$ \\
\hline Sexo & Feminino & $9(60,0 \%)$ & $42(45,2 \%)$ \\
\hline Etnia & Branca & $14(93,3 \%)$ & $91(97,8 \%)$ \\
\hline Idade & Em anos & $45,6( \pm 9,9)$ & $41,3( \pm 11,4)$ \\
\hline IES & Pública & $8(53,3 \%)$ & $57(61,3 \%)$ \\
\hline Tempo formado & Em anos & $22,0( \pm 11,3)$ & $18,2( \pm 11,8)$ \\
\hline Pós-graduação & Concluída & $9(60,0 \%)$ & $79(84,9 \%)$ \\
\hline Maior titulação & Aperfeiçoamento & $1(11,1 \%)$ & $5(6,3 \%)$ \\
\hline & Especialização & $8(88,9 \%)$ & $52(65,8 \%)$ \\
\hline & Mestrado & - & $9(11,4 \%)$ \\
\hline & Doutorado & - & $13(16,5 \%)$ \\
\hline Áreas & Endodontia & & $19(23,8 \%)$ \\
\hline & Saúde coletiva & $5(55,6 \%)$ & $13(16,3 \%)$ \\
\hline & Periodontia & - & $14(17,5 \%)$ \\
\hline & Cirurgia & - & $12(15,0 \%)$ \\
\hline & Estomatologia & - & $6(7,5 \%)$ \\
\hline & PNE\& & - & $3(3,8 \%)$ \\
\hline & Outras & $5(61,1 \%)$ & $33(41,5 \%)$ \\
\hline
\end{tabular}

$\mathrm{dp}^{*}=$ desvio-padrão; IES = Instituição de Ensino Superior $\mathrm{PNE}^{\&}=$ paciente portador de necessidades especiais

Na capital, o vínculo empregatício foi exclusivamente do tipo estatutário e CLT, já no interior do Estado, este tipo de vinculação caiu para 56,8\%. Em relação à capacitação prévia, os participantes informaram que foram desenvolvidas pelo gestor estadual, DATASUS ou por Universidades. Parte expressiva dos profissionais já atuou na APS e, comparado a ela, considerou o CEO um lugar privilegiado, indicando como o principal motivo (34,3\%) "aspectos relacionados com a finalidade do CEO” (Tabela 2 ).

Tabela 2 - Características das condições de trabalho, CEOs, RS, 2010

\begin{tabular}{|c|c|c|c|}
\hline \multicolumn{2}{|c|}{ Variável } & \multirow{2}{*}{$\begin{array}{c}\begin{array}{c}\text { Coordenadores } \\
\mathrm{N} / \%(\mathrm{~N}=15) \\
\mathrm{Md}^{\mathrm{C}}\left(\mathrm{P}^{\mathrm{a}} 25-\mathrm{P} 50\right)\end{array} \\
8(53,3 \%)\end{array}$} & \multirow{2}{*}{$\begin{array}{c}\text { Dentistas } \\
\mathrm{N} / \%(\mathrm{~N}=93) \\
\mathrm{Md}^{\mathrm{C}}(\mathrm{Pa} 25-\mathrm{P} 50) \\
44(47,3 \%)\end{array}$} \\
\hline Vínculo & Concursado & & \\
\hline & CLT & $4(26,7 \%)$ & $17(18,3 \%)$ \\
\hline & Terceirizado & $3(20,0 \%)$ & $11(11,8 \%)$ \\
\hline & Outros & - & $21(22,6 \%)$ \\
\hline \multirow[t]{3}{*}{$\mathrm{CH}^{\top}$ semanal } & Até 20 & $5(38,5 \%)$ & $44(47,8 \%)$ \\
\hline & 30 & $3(23,1 \%)$ & $12(13,0 \%)$ \\
\hline & 36 a 44 & $5(38,4 \%)$ & $36(39,2 \%)$ \\
\hline Salário & & $\begin{array}{r}3500 \\
(2200-5000)\end{array}$ & $\begin{array}{r}2424 \\
(1500-5000)\end{array}$ \\
\hline \multirow[t]{6}{*}{ Área de atuação } & Endodontia & $3(30,0 \%)$ & $32(34,4 \%)$ \\
\hline & Atenção PNE & $4(40,0 \%)$ & $22(23,7 \%)$ \\
\hline & Periodontia & $2(20,0 \%)$ & $20(21,5 \%)$ \\
\hline & CTBMF & $3(30,0 \%)$ & $16(17,2 \%)$ \\
\hline & Estomatologia & $2(20,0 \%)$ & $15(16,1 \%)$ \\
\hline & Outras & $4(40,0 \%)$ & $26(28,1 \%)$ \\
\hline $\begin{array}{l}\text { Atuação prévia } \\
\text { APS }^{\infty}\end{array}$ & Sim & $13(92,9 \%)$ & $51(57,3 \%)$ \\
\hline $\begin{array}{l}\text { Local } \\
\text { privilegiado }\end{array}$ & Sim & $10(76,9 \%)$ & $37(72,5 \%)$ \\
\hline
\end{tabular}

RFO, Passo Fundo, v. 22, n. 1, p. 43-48, jan./abr. 2017
A maioria dos profissionais informou conhecer as metas mínimas mensais para o CEO e as considerou adequadas. Setenta e um participantes $(73,2 \%)$ confirmaram a existência de exigência de cumprimento de produção no serviço. Para $37,6 \%$ dos CDs o número de pacientes atendidos por turno é de, no máximo, 4 usuários.

Há um número restrito de participantes que atuam sempre com auxiliar de saúde bucal (29\%). Sessenta e cinco profissionais $(60,7 \%)$ não reconhecem programa de educação permanente nas instituições, mas informam acesso a colegas para discussão de casos. Poucos participantes $(11,2 \%)$ relataram presença em fóruns de participação, os espaços referidos foram Conselhos de Saúde e os vinculados à área de exercício profissional. A maioria dos participantes, $83(80,6 \%)$, trabalha em outro local além do CEO; $56,8 \%$ com jornada de trabalho superior a 40 horas por semana; $47,8 \%$ dos profissionais relataram renda total entre 5 e 10 salários mínimos de referência (Tabela 3 ).

Tabela 3 - Características das relações de trabalho, CEOs, RS, 2010

\begin{tabular}{|c|c|c|c|}
\hline \multicolumn{2}{|c|}{ Variável } & \multirow{2}{*}{\begin{tabular}{|r|}
$\begin{array}{c}\text { Coordenadores } \\
\mathrm{N} / \%(\mathrm{~N}=15) \\
\text { média }\left( \pm \mathrm{dp}^{*}\right)\end{array}$ \\
$4(40,0 \%)$
\end{tabular}} & \multirow{2}{*}{\begin{tabular}{|c|}
$\begin{array}{c}\text { Dentistas } \\
\mathrm{N} / \%(\mathrm{~N}=93) \\
\left.\text { média }( \pm \mathrm{dp})^{*}\right) \\
25(27,8 \%)\end{array}$ \\
\end{tabular}} \\
\hline Atua com $\mathrm{ASB}^{\mathrm{D}}$ & Sempre & & \\
\hline & Quase sempre & $1(10,0 \%)$ & $13(14,4 \%)$ \\
\hline & Esporadicamente & $3(30,0 \%)$ & $22(24,4 \%)$ \\
\hline & Quase nunca & - & $12(13,3 \%)$ \\
\hline & Nunca & $2(20,0 \%)$ & $18(20,0 \%)$ \\
\hline \multirow[t]{2}{*}{ Capacitação } & Coordenação & $5(35,7 \%)$ & \\
\hline & Clínica & $2(20,0 \%)$ & $13(15,9 \%)$ \\
\hline Discussão & Sim & $10(100 \%)$ & $86(93,5 \%)$ \\
\hline EPb na instituição & Não & $11(73,3 \%)$ & $54(58,7 \%)$ \\
\hline $\begin{array}{l}\text { Frequenta fórum } \\
\text { de participação? }\end{array}$ & Não & $11(73,3 \%)$ & $84(91,3 \%)$ \\
\hline $\begin{array}{l}\text { Espaço para } \\
\text { sugestões/ } \\
\text { reclamações }\end{array}$ & Sim & $11(73,3 \%)$ & $49(54,4 \%)$ \\
\hline Outro trabalho & Sim & $10(83,3 \%)$ & $73(80,2 \%)$ \\
\hline $\mathrm{CH}^{\top}$ Total & & $46,0( \pm 15,0)$ & $47,9( \pm 9,4)$ \\
\hline Problemas de saúde & Sim & $4(33,3 \%)$ & $22(23,7 \%)$ \\
\hline
\end{tabular}

Os elementos estruturais e relacionais no ambiente de trabalho do CEO foram avaliados pela maioria dos profissionais como bons. Mas, para tamanho da equipe de pessoal auxiliar e salário, a soma dos resultados, "regular" e "ruim e muito ruim" representou $55,3 \%$ e $58,5 \%$, das respostas. O grau de satisfação com o trabalho no CEO foi alto $(68,3 \%)$.

\section{Discussão}

O reconhecimento do perfil dos profissionais, da sua visão sobre o espaço e as relações de trabalho pode auxiliar na compreensão da constituição e da dinâmica do serviço, favorecendo o entendimento do 
desempenho da organização. Este estudo revelou, nos CEOs do RS, um grupo de profissionais equilibradamente distribuídos segundo o sexo, com idade e tempo de graduação que sugerem experiência profissional e alta qualificação. A maior parte dos trabalhadores possuía vínculo empregatício formal, com indicação de permanência no serviço público. Houve avaliação positiva das condições e relações de trabalho, alto grau de satisfação, mas considerações negativas sobre aspectos salariais e dimensionamento de pessoal auxiliar.

Os dados sobre os CEOs em funcionamento no RS confirmam a distribuição não homogênea, com baixa cobertura populacional e baixo número de novas implantações destes serviços ${ }^{11}$. As razões que dificultam a expansão de CEOs nos municípios do RS podem estar relacionadas com o desempenho econômico do RS nas duas últimas décadas e déficit das contas públicas, às exigências estabelecidas pela lei de responsabilidade fiscal e a critérios estaduais de habilitação mais rigorosos para o $\mathrm{CEO}$ até $2009^{11,12}$. Já na modalidade de CEO, a maior frequência de CEOs do tipo 1 não acompanhou os resultados encontrados em estudos no Brasil ${ }^{13,14,15}$ onde os CEOs de modalidade tipo 2 são os de maior expressão. Considerando que melhores resultados parecem estar associados ao CEO tipo $3^{13,15}$, é importante observar a ausência deste tipo de CEO no RS até 2010.

Dentre as características sócio demográficas, observou-se um equilíbrio entre os sexos, o que acompanhou o resultado do RS no estudo do perfil de formação de 2010 de Morita et $a{ }^{16}(2010)$, e se distinguiu de estudos de outros serviços de saúde, de outras regiões, onde se observa tendência a maior participação do sexo feminino ${ }^{17,18,19,20}$. Resultado expressivo foi obtido através da etnia, com a presença de uma maioria de profissionais brancos $(97,2 \%)$, similar ao encontrado por Funk ${ }^{21}$ (2004) (95\%). A unidade étnica na formação e na constituição da força de trabalho encontrada é condizente com os estudos sobre desigualdade social no acesso à educação com recorte étnico/racial e vem sendo apontada como uma barreira cultural no cuidado em saúde $22,23,24,25$.

A maioria dos CDs dos CEOs RS provinha de Universidades públicas, o que contraria a tendência da formação brasileira onde há maior percentual de formados em Universidades privadas. Houve alta qualificação da força de trabalho, sendo a titulação de maior expressão a de especialista na área de endodontia. Resultados similares foram encontrados nos CEOs da Região de Saúde da Grande $\mathrm{Natal}^{26}$. A região Sul é a segunda região do Brasil em número de especialistas em Odontologia com 21,3\%, e isso se reflete nos CEOs do $\mathrm{RS}^{16}$. Estudos indicam a área de endodontia como a terceira de maior inscrição como especialista no RS e segunda no Brasil ${ }^{16,27}$. Além disso, a obrigatoriedade de determinadas áreas no CEO torna a presença de pós-graduação nestas áreas mais provável, ainda que não exista tal exigência para atuação no serviço. A alta presença de endodontistas aliado à alta ocupação nesta área poderia explicar o efeito superior de produção em endodontia em municípios com CEO encontrados em outros estudos ${ }^{15,28}$. Das áreas obrigatórias, verificou-se que a formação específica em atenção ao portador de necessidades especiais é a mais rara. Até 2007, Paranhos et al. ${ }^{27}$ (2009) relataram a presença de somente 19 profissionais com inscrição no Conselho Federal de Odontologia nesta área no RS, o que corrobora a perspectiva de carência destes profissionais. Entretanto, é preciso considerar que esta especialidade faz parte de um grupo mais recente na Odontologia, que só poderia ser registrada depois de 2002.

Nas relações trabalhistas, os resultados apontaram para a formalidade, através de formas diretas de contratação, como concursado/estatutário e CLT. Isto contrasta com a alta frequência de formas precárias de relações de trabalho dos CDs na ESF no Brasil encontrada em 2008 (51,7\%), mas estão de acordo com o fator regional, uma vez que esta precarização se reduz na análise por região, para a região $\mathrm{Sul}^{29}$. A maior informalidade desta relação nos CEOs do interior acompanhou a tendência de informalidade na condução das relações de trabalho verificada por Nogueira ${ }^{9}$. Na comparação com outros estudos sobre o CEO, verificou-se que na região da Grande Natal, $14 \%$ foram selecionados via concurso, e no Brasil 35,7\% dos CDs não eram concursados $^{14,16}$.

Uma das principais insatisfações encontradas foi relacionada ao salário pago pelo $\mathrm{CEO}$, que pode ser reflexo do declínio dos valores salariais nos últimos anos. Segundo o Departamento Intersindical de Estatística e Estudos Socioeconômicos ${ }^{30}$, os salários dos ocupados em serviços de saúde entre 1988 e 2008 apresentam tendências de declínio em todas as áreas de saúde, o que contribui para o aumento de trabalhadores com dupla jornada, e a extensão da jornada média para além das 44 horas. A maior parte dos profissionais relatou trabalhar em outro local e atuava em média 47 horas semanais $( \pm 10,1)$, tempo superior ao obtido por Michel-Crossato ${ }^{18} \mathrm{em}$ que CDs da Prefeitura Municipal de São Paulo trabalhavam em média 37,3 horas. $\mathrm{O}$ impacto do trabalho na saúde dos profissionais encontrado foi baixo perto do observado por outros estudos ${ }^{17,18}$, mas foi compatível com o tipo de problema ${ }^{18}$.

Os fracos resultados sobre a capacitação prévia às atividades no CEO acompanharam os achados de estudos na região da Grande Natal e no Brasil ${ }^{14,16}$. Este resultado, associado ao escasso relato de presença de educação permanente na instituição, parece evidenciar que há baixo acesso para a maioria dos profissionais a alguma forma de reflexão sobre o trabalho no CEO. O fato de que $32,8 \%$ dos profissionais desconhecem as metas mínimas estabelecidas pelo MS para o serviço pode ser um indicador da 
ausência de reflexão sobre o trabalho no CEO. Por outro lado, houve relato de acesso a outros profissionais para discussão de casos $(94,1 \%)$, e participação em reuniões de equipe (94,7\%), o que favorece a discussão e qualifica o processo de trabalho.

Em relação à percepção dos profissionais sobre os elementos estruturais e relacionais do CEO, verificou-se um bom julgamento para quase todos os quesitos. Esta percepção é parcialmente corroborada por um estudo, em Resende, de um Programa de Saúde do Adolescente, onde foi constatada insatisfação quanto ao ambiente físico e equipamentos e em relação às condições salariais, mas boa relação com os colegas e a chefia imediata ${ }^{31}$. Nos CEOs RS houve insatisfação com o dimensionamento de pessoal auxiliar. Este dado, associado ao fato de que $57,0 \%$ dos profissionais quase nunca atuavam com pessoal auxiliar, pode indicar o não atendimento quanto ao número de auxiliares disposto na legislação do CEO.

A logística da coleta permitiu o alcance da grande maioria dos profissionais, porém, por ser um questionário auto preenchido, houve perdas em algumas questões. Alguns CEOs recebem estagiários de Odontologia, a ausência destes no estudo e a participação de professores/preceptores pode ter gerado viés nos resultados.

\section{Conclusão}

Os resultados deste estudo permitiram traçar o perfil dos profissionais dos CEOs do RS, suas condições e relações de trabalho e seu grau de satisfação, constatando-se alta qualificação, boas condições estruturais e de relações interpessoais e alto grau de satisfação dos trabalhadores, que constituem indicações de um ambiente interno favorável ao bom desempenho dos CEOs. Entretanto, algumas fragilidades foram observadas, como as condições de vinculação de trabalho no interior do Estado, as condições salariais, as atividades de capacitação, ações de educação permanente e o dimensionamento da equipe de pessoal auxiliar e a similaridade étnica. Maiores estudos precisam ser realizados sobre o dimensionamento, o processo de trabalho e de educação permanente destas equipes, suas expectativas e motivações com o trabalho no CEO.

\section{Abstract}

Objective: to outline the profile and describe the perception of professionals on working conditions and relationships, and the level of satisfaction when working at Dental Specialty Centers in the state of Rio Grande do Sul, Brazil. Subjects and method: 108 professionals from 17 Dental Specialty Centers of Rio Grande do Sul participated in the study and answered a structured questionnaire. Results: the results revealed the presence of a group that was evenly distributed according to gender, with average age of 41.9 years $( \pm 11.2)$, prevalence of white ethnicity, high frequency of post-graduated individuals (81.5\%), and higher rate of formal employment contracts (67.6\%). Most elements regarding working conditions and relationships were positively evaluated, except for salary and size of dental hygienist teams. The level of satisfaction with the work in Dental Specialty Centers was high. Conclusion: the results suggest a positive relation between professionals and their work environment. The weakest elements were conditions of employment contracts in upstate regions, salary conditions, training activities, actions for continued education, and the size of dental hygienist teams.

Keywords: Secondary healthcare. Healthcare. Health personnel.

\section{Referências}

1. Brasil sorridente leva tratamento odontológico para $80 \mathrm{mi}-$ lhões de pessoas. Portal Brasil. [Acessado 2017 abr 18]. Disponível em: http://www.brasil.gov.br/governo/2014/06/brasil-sorridente-leva-tratamento-odontologico-para-80-milhoes-de-pessoas

2. Brasil. Ministério da Saúde, Secretaria de Atenção à Saúde, Departamento de Atenção Básica, Coordenação Nacional de Saúde Bucal. Diretrizes da Política Nacional de Saúde Bucal 2004 Jan. [Acessado 2017 abr 18]. Disponível em: http:// bvsms.saude.gov.br/bvs/publicacoes/politica_nacional_brasil_sorridente.pdf

3. Brasil. Portaria 599/GM de 23 de março de 2006. Define a implantação de Especialidades Odontológicas (CEOs) e de Laboratórios Regionais de Próteses Dentárias (LRPD), e estabelece critérios, normas e requisitos para seu financiamento. Diário Oficial da União 2006; 24 de mar.

4. Brasil. Portaria 600/GM de 23 de março de 2006. Institui o financiamento dos Centros de Especialidades Odontológicas. Diário Oficial da União 2006; 24 de mar.

5. Brasil. Portaria 2898/GM de 21 de setembro de 2010. Atualiza o anexo da Portaria 600/GM de 23 de março de 2006. Diário Oficial da União 2010; 23 de set.

6. Brasil. Portaria 1032/GM de 5 de maio de 2010. Inclui procedimento odontológico na Tabela de Procedimentos, Medicamentos, Órteses e Próteses e Materiais Especiais do Sistema Único de Saúde - SUS, para atendimento às pessoas com necessidades especiais. Diário Oficial da União 2010; 06 de maio.

7. Brasil. Portaria 718/GM de 20 de dezembro de 2010. Altera a estrutura da tabela, exclui, inclui, e compatibiliza procedimento odontológico na Tabela de Procedimentos, Medicamentos, Órteses e Próteses e Materiais Especiais do Sistema Único de Saúde - SUS. Diário Oficial da União 2010; 31 de dez.

8. Brasil. Retificação na Portaria 718/GM de 20 de dezembro de 2010. Diário Oficial da União 2011; 08 fev.

9. Nogueira R, coordenador. Avaliação de tendências e prioridades sobre recursos humanos de saúde. Rede Observatório de Recursos Humanos em Saúde, Organização Pan-Americana de Saúde. Brasília; 2002.

10. Brasil. Ministério da Saúde, Secretaria de Atenção à Saúde, Departamento de Regulação, Avaliação e Controle de Sistemas, Coordenação Geral de Regulação e Avaliação. Programa Nacional de Avaliação dos Serviços de Saúde - PNASS. 2004. [Acessado 2017 abr 18]. Disponível em http://www.anvisa.gov.br/servicosaude/avalia/pnass.pdf 
11. Rio Grande do Sul (Estado). Secretaria de Saúde. Política de Saúde Bucal do Rio Grande do Sul. [Acessado 2017 abr 18]. Disponível em: http://www.saude.rs.gov.br/upload/1337629466_ Pol\%C3\%ADtica\%20de\%20Sa\%C3\%BAde\%20Bucal.pdf

12. Fundação de Economia e Estatística. Resumo Estatístico RS - 2011. Panorama Recente do Desenvolvimento Econômico do RS. FEE; 2011. [Acessado 2017 abr 18]. Disponível em http://cdn.fee.tche.br/resumo/resumo-rs-2011_site.pdf

13. Figueiredo N. Centros de Especialidades Odontológicas CEO: um estudo linha de base para avaliação e monitoramento dos serviços para a região Nordeste [Tese de Doutorado]. Pernambuco: Universidade Federal de Pernambuco; 2008.

14. Deitos AR. Avaliação da atenção especializada em saúde bucal [Dissertação de Mestrado]. São Paulo: Universidade de São Paulo; 2009.

15. Celeste RK, Moura FR, Santos CP, Tovo MF. Analysis of outpatient care in Brazilian municipalities with and without specialized dental clinics, 2010. Cad Saude Publica 2014; 30(3):511-21.

16. Morita MC, Haddad AE, Araújo ME. Perfil atual e tendências do cirurgião-dentista brasileiro. Rede Observatório de Recursos Humanos em Saúde. Organização Pan-Americana de Saúde. Maringá: Dental Express, 2010. [Acessado 2017 abr18]. [cerca de 96p]. Disponível em http://cfo.org.br/wp-content/uploads/2010/04/PERFIL_CD_BR_web.pdf

17. Nunes MF, Freire MCM; Qualidade de vida de cirurgiões-dentistas que atuam em um serviço público. Rev Saúde Pública 2006; 40(6):1019-26.

18. Michel-Crosato E. Perfil da força de trabalho representada pelo cirurgião-dentista: análise epidemiológica dos profissionais que exerciam suas atividades na Prefeitura Municipal de São Paulo [Tese de Doutorado]. São Paulo(SP): Universidade de São Paulo; 2008.

19. Araújo YP, Dimenstein M. Estrutura e organização do trabalho do cirurgião-dentista no PSF de municípios do Rio Grande do Norte. Cienc Saúde Colet 2006;11(1);219-27.

20. Martinelli PJL, Macedo CSLV, Medeiros KJ, Silva SF, Cabral APS, Pimentel FC, et al. Perfil do cirurgião-dentista inserido na Estratégia de Saúde da Família em municípios do estado de Pernambuco, Brasil. Cienc Saúde Colet 2010; 15(Supl 2):3243-8

21. Funk PP, Flôres MMDZ, Garbin CA, Hartmann MSM, Mendonça JL. Perfil do profissional formado pela Faculdade de Odontologia da Universidade de Passo Fundo/RS: da formação à realidade profissional. Passo Fundo 2004; 9(2):105-9.

22. Mitchell DA, Lassiter SL. Addressing Health Care Disparities and Increasing Workforce Diversity: The Next Step for the Dental, Medical, and Public Health Professions. Am J Public Health 2006; 96(12):2093-7.

23. Costa BM. Aspectos da desigualdade racial em Porto Alegre. Observatório da cidade de Porto Alegre. [Acessado 2017 abr18]. Disponível em: http://proweb.procempa.com.br/ pmpa/prefpoa/observatorio/usu_doc/texto_raca_etnia_3.pdf

24. Silva NV, Hasenbalg C. Tendências da desigualdade educacional no Brasil. Dados 2000; V 43(3):423-45

25. Bittar M, Almeida CEM. Mitos e controvérsias sobre a política de cotas para negros na educação superior. Educ Rev 2006; 28:141-59.

26. Souza GCA. Centros de Especialidades Odontológicas: avaliação da atenção em média complexidade na rede pública da região da grande Natal [Dissertação de Mestrado]. Natal (RN): Universidade Federal do Rio Grande do Norte; 2009.
27. Paranhos LR, Ricci ID, Scanavini MC, Bérzin F, Ramos AL. Análise do Mercado de Trabalho Odontológico na Região Sul do Brasil. Ver Fac Odontol Univ Passo Fundo 2009; 14(1):7-13.

28. Maia LS, Kornis GEM. A reorganização da atenção à saúde bucal frente aos incentivos federais: a experiência fluminense. Rev APS 2010; 13(1):84-95.

29. Taveira ZZ. Precarização dos vínculos de trabalho na estratégica da saúde da família: revisão de literatura [Monografia]. Minas Gerais (MG): Universidade Federal de Minas Gerais; 2010.

30. Ministério do Trabalho e Emprego (Brasil). Fundo do Amparo ao Trabalhador. Fundação Sistema Estadual de Análise de Dados. Departamento Intersindical de Estatística e Estudos Socioeconômicos - DIEESE. O trabalho na saúde - 19982008. Boletim Trabalho na Saúde 2009; (1):1-11.

31. Afonso LM, Rocha HM. Fatores organizacionais que geram insatisfação no servidor público e comprometem a qualidade dos serviços prestados. VII Simpósio de Excelência em Gestão e Tecnologia 2010; Rio de Janeiro.

\section{Endereço para correspondência:}

Karla Frichembruder

UFRGS/Faculdade de Odontologia/CPOS

Ramiro Barcelos, 2492, sala 402A

CEP 90035-003 Cidade Porto Alegre, RS, Brasil

Fone: 05133085204

E-mail: kfrichem@gmail.com

Recebido: 14/11/2016. Aceito: 02/03/2017. 